\title{
Human-monoclonal-antibody therapy protects nonhuman primates against advanced Lassa fever
}

\author{
Chad E Mire ${ }^{1,2,11(D)}$, Robert W Cross ${ }^{1,2,11}$, Joan B Geisbert ${ }^{1,2}$, Viktoriya Borisevich ${ }^{1,2}$, Krystle N Agans ${ }^{1,2}$ (1), \\ Daniel J Deer ${ }^{1,2}$, Megan L Heinrich ${ }^{3}$, Megan M Rowland ${ }^{3}$, Augustine Goba ${ }^{4,5}$, Mambu Momoh ${ }^{4-6}$, \\ Mathew L Boisen ${ }^{3}$, Donald S Grant ${ }^{4,5}$, Mohamed Fullah ${ }^{4,5,10}$, Sheik Humarr Khan ${ }^{4,5,10}$, Karla A Fenton ${ }^{1,2}$, \\ James E Robinson ${ }^{7}$, Luis M Branco ${ }^{3,11}$, Robert F Garry ${ }^{3,8,9}$ \& Thomas W Geisbert $^{1,2}$
}

\begin{abstract}
There are no approved treatments for Lassa fever, which is endemic to the same regions of West Africa that were recently devastated by Ebola. Here we show that a combination of human monoclonal antibodies that cross-react with the glycoproteins of all four clades of Lassa virus is able to rescue $100 \%$ of cynomolgus macaques when treatment is initiated at advanced stages of disease, including up to $8 \mathrm{~d}$ after challenge.
\end{abstract}

Lassa virus (LASV) infects more than 300,000 individuals per year across West Africa, causing over 3,000 deaths ${ }^{1}$. The case-fatality rate (CFR) for the hemorrhagic fever Lassa fever (LF) has historically ranged from $15 \%$ to $50 \%$ in hospitalized patients, and a recent multi-year study in Sierra Leone reported a 69\% CFR in admitted patients ${ }^{2}$. Currently, a large outbreak of LF that began in August of 2015 is devastating Nigeria. Between December 2016 and June 2017, the World Health Organization reported a total of 501 suspected cases (175 laboratory confirmed) and 59 deaths among all cases, with a CFR of $33.7 \%{ }^{3}$. Previous and recent importation of LF into Europe and the US by travelers on commercial airlines ${ }^{4,5}$ illustrates the potential for spread outside the endemic area. Moreover, LASV is also classified by several US government agencies as a 'Category A' priority pathogen because of a concern about deliberate misuse of the virus.

So far, no preventive vaccines or therapeutics for LF have been approved for human use, and only a few candidate vaccines and treatments, such as ribavirin, have been assessed in preclinical studies for their potential to protect against against wild-type LASV in nonhuman primates (NHPs) ${ }^{6-10}$. Intravenous ribavirin has been shown to reduce the mortality from $55 \%$ to $5 \%$ in individuals at high risk for the disease who present with elevated liver enzymes upon hospital admission, provided that the treatment is administered during the first $6 \mathrm{~d}$ of illness ${ }^{11}$. There are also a few reports of convalescent plasma being administered to individuals with LASV infection ${ }^{12-14}$, and in one of these cases, a beneficial effect was observed when the plasma was administered early in the disease course ${ }^{14}$. Notably, it was shown that immune plasma protected cynomolgus monkeys if given shortly after LASV exposure and if the plasma contained neutralizing antibodies that were closely matched to the challenge strain of LASV ${ }^{10}$. These neutralizing antibodies were found to bind the LASV glycoprotein (GP), which is composed of the GP subunits GP1 and GP2, and either (i) prevent the binding of the GP1 subunit to the receptor $\alpha$-dystroglycan ${ }^{15}$; (ii) prevent fusion of the viral envelope with the host-cell membrane through GP2-subunit binding; or (iii) perform a combination of both. These NHP data suggested that antibodies targeting the GP of LASV might be of clinical benefit for individuals with LF, and that they thus warranted further preclinical study of antibody efficacy against LASV.

Here we used the cynomolgus macaque model of lethal LASV to assess the protective efficacy of five promising LASV-GP-neutralizing huMAbs $(37.2 \mathrm{D}, 12.1 \mathrm{~F}, 8.9 \mathrm{~F}, 19.7 \mathrm{E}$, and $37.7 \mathrm{H})$ on the basis of crossreactivity to the four clades (I-IV) of LASV GP, neutralization from in vitro assays ${ }^{16}$, and protective efficacy in guinea pigs ${ }^{17}$. Macaques were inoculated on day 0 with a target dose of 3,500 plaque-forming units (PFU), and these huMAbs were administered as a monotherapy referred to as a single-antibody treatment (STx) cohort (Fig. 1a); treatment was initiated at day 0 after LASV exposure, with additional treatments administered during the course of infection for all STx cohorts (19.7E, at day 0 and 5 postinfection (p.i.) $(n=4) ; 37.2 \mathrm{D}$, day 0,4 , and 8 p.i. $(n=6) ; 12.1 \mathrm{~F}$, day 0,4 , and 8 p.i. $(n=3) ; 37.7 \mathrm{H}$, day 0,4 , and 8 p.i. $(n=3)$; or $8.9 \mathrm{~F}$, day 0,4 , and 8 p.i. $(n=4))$. Untreated control animals $(n=7)$ had a mean time to death of 12 d p.i. (Fig. 1a; Supplementary Table 1, bracket denotes which experiment each control is from); clinical signs consistent with LF (Supplementary Table 1); and viremia, as measured by infectious LASV (Fig. 1b) and RT-qPCR (Supplementary Fig. 1a) as early as days 4 and 3 p.i., respectively. Each huMab was well tolerated, and all STX-huMAb treatment regimens resulted in both $100 \%$ protection

\footnotetext{
${ }^{1}$ Department of Microbiology and Immunology, University of Texas Medical Branch at Galveston, Galveston, Texas, USA. ${ }^{2}$ Galveston National Laboratory, University of Texas Medical Branch at Galveston, Galveston, Texas, USA. ${ }^{3}$ Zalgen Labs, Germantown, Maryland, USA. ${ }^{4}$ Viral Hemorrhagic Fever Program, Kenema Government Hospital, Kenema, Sierra Leone. ${ }^{5}$ Ministry of Health and Sanitation, Freetown, Sierra Leone. ${ }^{6}$ Polytechnic College, Kenema, Sierra Leone. ${ }^{7}$ Sections of Infectious Disease, Departments of Pediatrics and Internal Medicine, School of Medicine, Tulane University, New Orleans, Louisiana, USA. ${ }^{8}$ Department of Microbiology and Immunology, Tulane University, New Orleans, Louisiana, USA. ${ }^{9}$ Tulane Center of Excellence, Global Viral Network, Tulane University, New Orleans, Louisiana, USA. ${ }^{10}$ Deceased. 11 These authors contributed equally to this work. Correspondence should be addressed to T.W.G. (twgeisbe@utmb.edu) or R.F.G. (rfgarry@tulane.edu).
} 

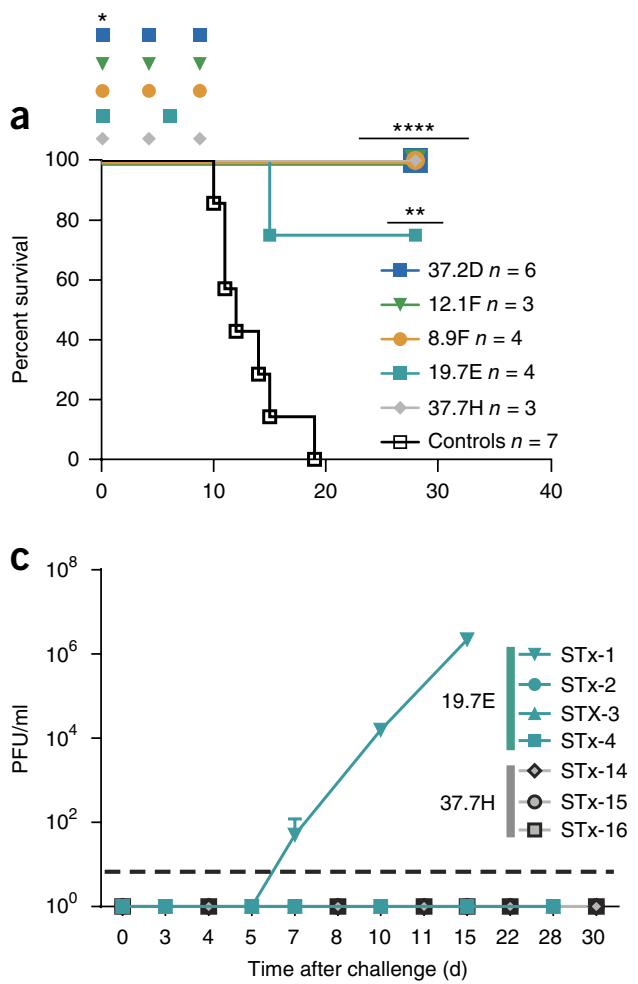

b
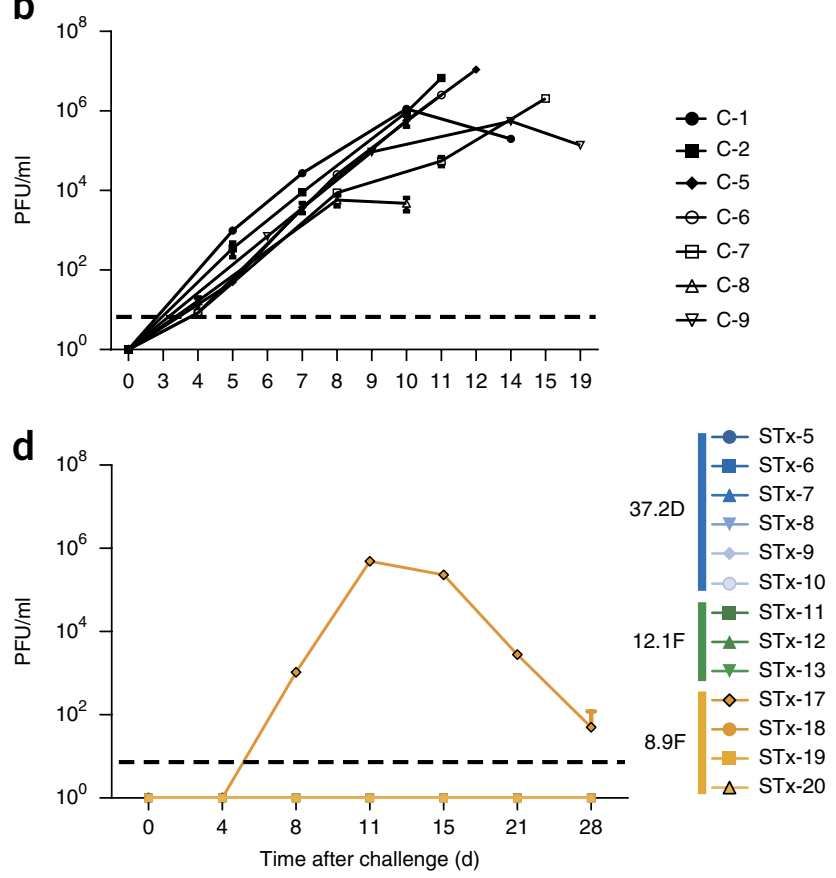

Figure 1 Protective efficacy profile of LASV GP huMAb monotherapy in a cynomolgus macaque model of lethal LF. (a) Kaplan-Meier survival curve for each cohort treated with a single anti-LASV GP huMAb (STx), beginning on day of LASV challenge. *Day of LASV challenge; huMAb symbols depicting the days of treatment post LASV challenge: blue square, 37.2D, days 0,4 , and 8 ; green triangle, $12.1 \mathrm{~F}$, days 0 , 4 , and 8 ; orange circle, $8.9 \mathrm{~F}$, days 0 , 4, and 8; teal square, 19.7E, days 0 and 5 ; gray diamond, $37.7 \mathrm{H}$, days 0,4 , and 8 ; and open squares, untreated controls. ${ }^{* *} P<0.0078$, ${ }^{* * * *} P<0.0001$. (star designation determined by GraphPad Prism 7.0 software); error bars are \pm s.d. from mean values, as determined by Mantel-Cox test. For b,c, and $\mathbf{d}$ : circulating infectious-virus load for each cohort, as measured by PFU/ml with the lower limit of detection of $15 \mathrm{PFU} / \mathrm{ml}$. C, control. STx, monotherapy huMAb treatment group. Error bars are \pm s.d. from mean values. Limit of detection is depicted as dashed line on graphs.

from LF and in survival to study endpoint (Fig. 1a; $P<0.0001$; Supplementary Table 2). One exception occurred in the STx cohort (Supplementary Table 2, STx-1-4) that received huMab 19.7E on days 0 and 5 after LASV exposure (Fig. 1a, $P<0.0078$ ), in which one of four in the cohort, animal STx-1, had detectable infectious virus (Fig. 1c) and viral RNA (Supplementary Fig. 1b) on days 5, 10, and 15 p.i. and experienced clinical signs of illness consistent with LF, requiring euthanasia (Supplementary Table 2). Animals STx-2-4 also had detectable viral RNA (Supplementary Fig. 1b), but none of the three had marked clinical pathology except for fever, which was detected in STx-4. Additionally, animal STx-17 in the 8.9F-treatment cohort (Supplementary Table 2, STx17-20) had detectable circulating infectious virus (Fig. 1d) and viral RNA (Supplementary Fig. 1c) on days $8,11,15,21$, and 28 p.i. (Fig. 1d); STx-20 from the 8.9F group and STx-13 from the 12.1F group (STx 11-13) had detectable viral RNA on day 8 p.i., with STx-20 and STx-13 clearing any detectable viral genomes by day 11 p.i. (Supplementary Fig. 1c).

We postulated that the circulating infectious viremia detected in animal STx-17 from the 8.9F-treatment cohort was due to an $8.9 \mathrm{~F}$ escape mutant, and we analyzed the sequence of the virus at day 21 p.i. Indeed, we isolated an $8.9 \mathrm{~F}$ escape mutant (LASV $8.9 \mathrm{~F}(\mathrm{e})$ ) from day 21 p.i. STx-17 serum that encoded a single amino acid mutation, Y150D, in the GP1 subunit (the only change found in the complete virus genome). The binding of $8.9 \mathrm{~F}$ to a glycoprotein complex (GPC) Y150D mutant expressed on the surface of HEK-293T/17 cells was abrogated, whereas binding by MAbs 37.2D and 12.1F was not reduced (Supplementary Table 3).
8.9F binding is conformation dependent, requiring both the GP1 and GP2 subunits, suggesting that this mutation affects the quaternary epitope required for $8.9 \mathrm{~F}$ binding 15,16 . Whereas LASV $8.9 \mathrm{~F}(\mathrm{e})$ escaped $8.9 \mathrm{~F}$ neutralization, this virus was completely neutralized in vitro by a cocktail (CTx) of 8.9F, 12.1F, and 37.2D (Supplementary Fig. 2). Although LASV mutants that develop resistance to a single antibody can be selected, we have yet to observe the emergence of resistant mutants in vitro and in vitro to these three antibodies in combination (data not shown), which suggests that a combination approach might have utility as a therapeutic modality.

To assess therapeutic combinations of these huMAbs, macaques were inoculated on day 0 with a target dose of 3,500 PFU, and these huMAbs were administered as a cocktail therapy referred to as CTx. Treatment was initiated at day $0(19.7 \mathrm{E}+37.2 \mathrm{D} ; n=4)$ and at days 3,6 , or $8(8.9 \mathrm{~F}+12.1 \mathrm{~F}+37.2 \mathrm{D} ; n=4,4$, and 5 , respectively) post LASV exposure (Fig. 2a; Supplementary Table 4). Untreated control animals $(n=6)$ had a mean time to death of $12 \mathrm{~d}$ p.i. (Fig. 2a; Supplementary Table 1, bracket denotes which experiment each control is from); clinical signs consistent with LF (Supplementary Table 1); and viremia, as measured by infectious LASV (Fig. 2b) and RT-qPCR (Supplementary Fig. 3a) as early as days 4 and 3 p.i., respectively. Each CTx treatment was well tolerated and resulted in $100 \%$ protection from LF and survival to study endpoint (Fig. 2a, $P<0.0001$; Supplementary Table 4). Of particular interest was the combination of the three neutralizing huMabs $8.9 \mathrm{~F}, 12.1 \mathrm{~F}$, and 37.2D, which, unlike the $19.7 \mathrm{E}+37.7 \mathrm{H}$ combination, react to distinct 

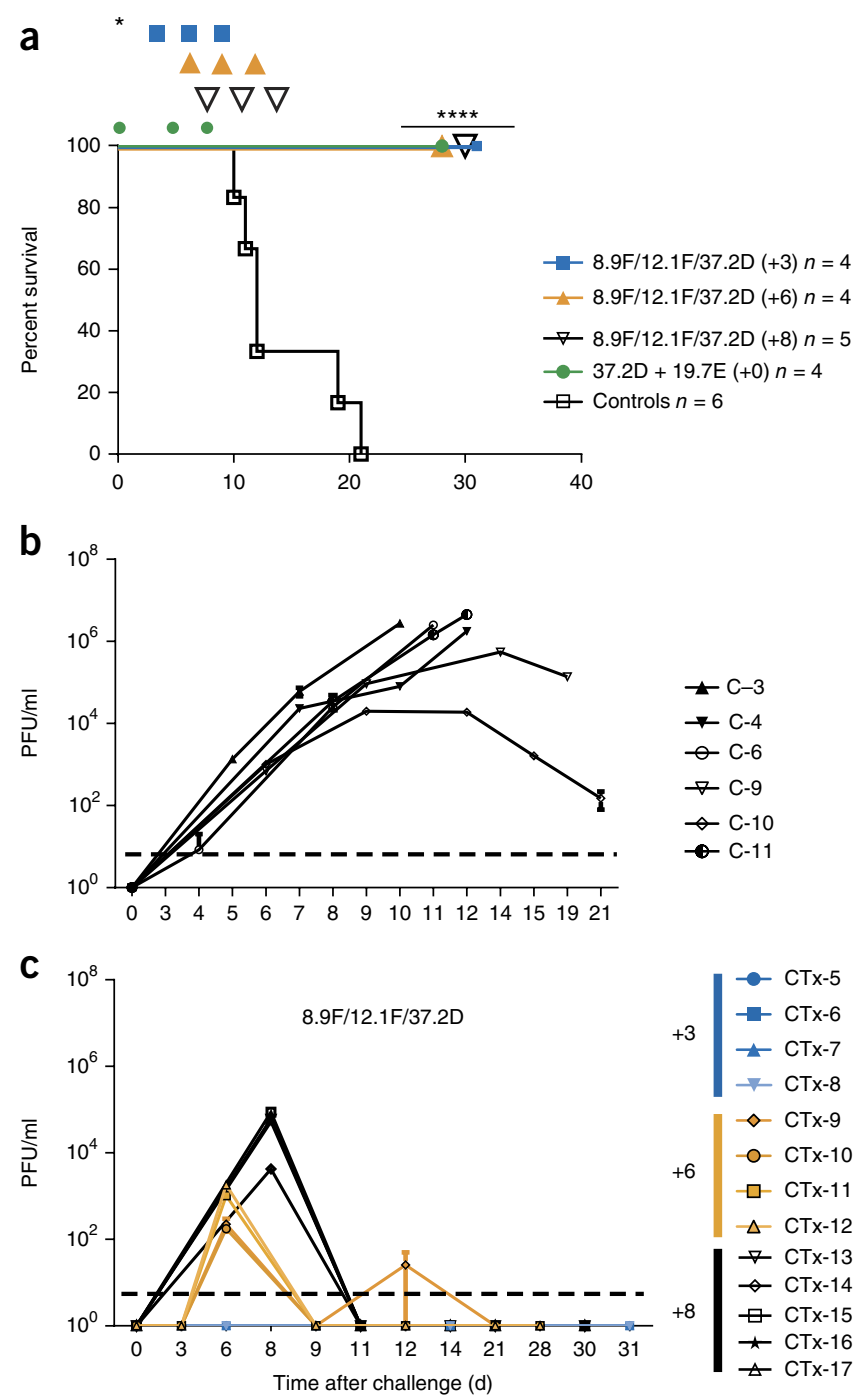

Figure 2 Protective-efficacy profile of LASV-GP huMAb combination therapy in a cynomolgus- macaque model of LF. (a) Kaplan-Meier survival curve for each cohort treated with a cocktail of anti-LASV-GP huMAb (CTx) after LASV challenge. $(37.2 \mathrm{D}+19.7 \mathrm{E})$ or day $3(+3)$, day $6(+6)$, or day $8(+8)$ post LASV challenge. *Day of LASV challenge; huMAb symbols depict the days of antibody-cocktail treatment post LASV challenge: blue square, +3 8.9F/12.F/37.2D cohort, days 3,6 , and 9; orange triangle, +6 8.9F/12.F/37.2D cohort, days 6,9 , and 12 ; open triangle, +8 8.9F/12.F/37.2D cohort, days 8,11 , and 14 ; and open squares, untreated controls. $* * * * P<0.0001$; as determined by Mantel-Cox test. In $\mathbf{b}$ and $\mathbf{c}$ : circulating infectious-virus load for each cohort, as measured by PFU/mI with the lower limit of detection of $15 \mathrm{PFU} / \mathrm{ml}$. C, control. CTx, cocktail-huMAb treatment group. Error bars are \pm s.d. from mean values. Limit of detection depicted as dashed line on graphs.

epitopes on the LASV-GP complex and bind the GP from four separate clades of LASV (clades I-IV), exhibiting the desirable potential for a cross-protective therapeutic for LASV in West Africa ${ }^{16}$. We used the $8.9 \mathrm{~F} / 12.1 \mathrm{~F} / 37.2 \mathrm{D}$ cocktail to assess the therapeutic efficacy in animals when treatment was initiated at an advanced stage of infection (Fig. 2a, days 6 and 8 p.i.), at which point animals were clinically ill (Supplementary Table 4, CTx-9-17) and had viremia (Fig. 2c, Supplementary Figs. 3b and 4). Animals from all three delayed CTx cohorts with treatment initiated at day $3(+3)$, day $6(+6)$, or day $8(+8)$ after LASV challenge were viremic, as assessed by detectable circulating
LASV genomes, on the first day of treatment (Supplementary Figs. 3b and 4). Notably, all animals in the +6 and +8 cohorts had detectable circulating infectious LASV on the initial day of treatment. Viremia resolved by day 9 or day 11 p.i., respectively, in these two cohorts, with a re-emergence of detectable infectious LASV in animal CTx-9 on day 12 (final treatment day) that resolved by day 14 p.i. (Fig. 2c). Additionally, major clinical signs and illness were resolved for all three delayed-treatment cohorts (Supplementary Table 4).

To assess the humoral immune response to LASV infection in the CTx cohorts treated with $8.9 \mathrm{~F} / 12.1 \mathrm{~F} / 37.2 \mathrm{D}$, the circulating levels of IgG to the LASV nucleoprotein (NP) (Supplementary Fig. 5a-d) were measured and compared to those of the control cohort. All treated animals had detectable levels of circulating anti-LASV-NP IgG, comparable to those observed in control animals. This suggests that the CTx therapy did not interfere with the humoral immune response to LASV infection.

At the study endpoint for each animal, tissues were examined by immunohistochemistry (IHC) for histopathology consistent with LF and for LASV antigen (Supplementary Figs. 6 and 7, and Supplementary Table 5). The control cohort after euthanasia had observable histopathology of the liver, spleen, and brain, as measured by perimembrane (liver) and diffuse cytoplasmic (spleen and brain (endothelial cells)) LASV immunolabeling. The CTx +6 and CTx +8 cohorts had no observable lesions beyond mild perivascular cuffing in the brain tissue (Supplementary Fig. 6k); no LASV immunolabeling was detected. However, LASV RNA was detectable in tissues at the study's endpoint (Supplementary Table 5), as has been observed in other viral hemorrhagic fever treatment and infection-model studies ${ }^{18,19}$. Three of the four animals from the CTx +3 cohort had no observable or scored lesions, as assessed by histology (Supplementary Fig. 7 and Supplementary Table 5, H\&E), and animal CTx-5 had minimal liver lesions and mild perivascular cuffing in the brain. Interestingly, only animal CTx-8 from this group had no observable LASV immunolabeling (Supplementary Fig. 7 and Supplementary Table 5; IHC); CTx-5, CTx-6, and CTx-7 were positive for LASV antigen in the liver, spleen, and brain (Supplementary Table 5, IHC).

We further investigated the viral-RNA and infectious-virus loads from LASV target organs of the +3 cohort, because this was the only treatment group that had LASV-antigen-positive tissues at the day-31 study endpoint. The control animal from this study, C-9 (day 19), was positive for LASV RNA in all tissues tested and had an infectious-virus load of greater than $6 \log _{10}$ PFU/g in the spleen; animal CTx-6 had a $3 \log$ lower infectious virus load in the spleen, whereas viral loads in tissues from CTx-5, CTx-7, and CTx-8 were below the limit of detection. Interestingly, there was no detectable infectious viremia in any animal from the $+3 \mathrm{CTx} 8.9 \mathrm{~F} / 12.1 \mathrm{~F} / 37.2 \mathrm{D}$ cohort, perhaps as a result of the anti-LASV MAbs still circulating, at all time points tested (Fig. $2 \mathrm{c}$ and Supplementary Figs. 3 and 4). Taken together, these data, in regard to NHP convalescence and virus detection, may not be novel, given that infectious LASV has been observed months after convalescence in the urine of humans who have recovered from the illness ${ }^{13}$. It is possible that the timing of the $8.9 \mathrm{~F} / 12.1 \mathrm{~F} / 37.2 \mathrm{D}$ combination treatment of the +3 cohort led to a cell-associated state for the virus that did not lead to detectable release of virus. It is also possible that the viruses had started to escape the organ, but not the local tissue environment into the circulation. Viral RNA in tissues could be associated with noninfectious virus, or it might be degraded. Initiating treatment at day +3 did not cause any marked cytopathology in the LASV-antigen-positive tissues, and there was no overt clinical pathology observed in this cohort at the study 


\section{BRIEF COMMUNICATIONS}

endpoint. The presence of LASV RNA in tissues in the $+3,+6$, and +8 cohorts raises the possibility that infectious virus could re-emerge at an extended time after treatment termination. It is also possible that infectious LASV may be present in immunologically privileged sites, such as the eyes or testes. These possibilities will be tested in future studies.

The current studies present a novel alternative for the treatment of LF infections, using an approach that relies on the use of fully human MAbs derived from mature B cells isolated from convalescent donors. Early studies that employed convalescent plasma to treat active LF infections met with mixed success. These results were likely the result of low titers of protective antibodies in donor blood, or specificities that were not well matched against circulating LASV strains or variants. The current approach, making use of either single injections or cocktails of rationally selected huMAbs, consistently conferred high-level protection from lethal LF in NHPs. These data support the advance of the huMAb 8.9F/12.1F/37.2D combination for further preclinical evaluation as a LF therapeutic.

\section{METHODS}

Methods, including statements of data availability and any associated accession codes and references, are available in the online version of the paper.

Note: Any Supplementary Information and Source Data files are available in the online version of the paper.

\section{ACKNOWLEDGMENTS}

The authors thank K. Schuenke (UTMB) for administrative assistance; N. Dobias (UTMB) and the University of Texas Medical Branch (UTMB) Research Histology Core for assistance with tissue preparations; and C. Klages, J. Graber, and the UTMB Animal Resource Center for veterinary and husbandry support. The authors also thank all the members of the Viral Hemorrhagic Fever Consortium for their continued support and contributions to the VHF field (http://www.vhfc.org). In memoriam: Tragically, two co-authors, who contributed greatly to public-health and VHF research efforts in Sierra Leone, contracted Ebola hemorrhagic fever and lost their battle with the disease before this manuscript could be published: Mohamed Fullah and Sheik Humarr Khan. We wish to honor their memory. This work was supported by the Department of Health and Human Services/National Institutes of Health/National Institute of Allergy and Infectious Diseases Challenge and Partnership Grant Numbers AI067188 (PI: R.F.G.) and AI082119 (PI: R.F.G.), Health and Human Services Contract HHSN272200900049C (PI: J.E.R.), and National Institutes of Health/National Institute of Allergy and Infectious Diseases grant number R01AI104621 (PI: R.F.G.), U19AI109762 (PI: E.O. Saphire),
UC7AI094660 to UTMB for BSL-4 operations support of the Galveston National Laboratory, and RC-0013-07 (PI: R.F.G.) from the Louisiana Board of Regents.

\section{AUTHOR CONTRIBUTIONS}

C.E.M., R.W.C., L.M.B, J.E.R., R.F.G., and T.W.G. conceived and designed the experiments. C.E.M., R.W.C., J.B.G., D.J.D., and T.W.G. performed the Lassa NHP challenge and treatment experiments and conducted clinical observations of the animals. J.B.G., K.N.A., and V.B. performed the clinical-pathology assays. J.B.G., R.W.C., and V.B. performed the LASV-infectivity assays. R.W.C. performed ELISA assays. C.E.M. and K.N.A. performed the PCR assays. C.E.M., R.W.C., L.M.B., J.B.G., V.B., K.N.A., D.J.D., M.M.R., M.L.H., A.G., M.M., D.S.G., K.A.F., J.E.R., R.F.G., and T.W.G. analyzed the data. K.A.F. performed histologic and immunohistochemical analysis of the data. M.L.B, A.G., M.M., M.F., R.W.C, L.M.B., and R.F.G. developed and validated LASV ELISA assays. S.H.K. and R.F.G managed the identification of convalescent LF patients and collected PBMCs for the derivation of huMAbs. J.E.R. and R.F.G. derived LASV huMAbs. C.E.M., R.W.C., L.M.B., R.F.G., and T.W.G. wrote the paper. All authors had access to all the data, and approved the final version of the manuscript.

\section{COMPETING FINANCIAL INTERESTS}

The authors declare competing financial interests: details are available in the online version of the paper.

Reprints and permissions information is available online at http://www.nature.com/ reprints/index.html. Publisher's note: Springer Nature remains neutral with regard to jurisdictional claims in published maps and institutional affiliations.

1. McCormick, J.B., Webb, P.A., Krebs, J.W., Johnson, K.M. \& Smith, E.S. J. Infect Dis. 155, 437-444 (1987).

2. Shaffer, J.G. et al. PLoS Negl. Trop. Dis. 8, e2748 (2014).

3. World Health Organization. Lassa Fever - Nigeria. Available at: http://www.who. int/csr/don/28-june-2017-lassa-fever-nigeria/en/. (Accessed 5 July 2017).

4. Schmitz, H. et al. Microbes Infect. 4, 43-50 (2002).

5. Amorosa, V. et al. Emerg. Infect. Dis. 16, 1598-1600 (2010).

6. Fisher-Hoch, S.P., Hutwagner, L., Brown, B. \& McCormick, J.B. J. Virol. 74 6777-6783 (2000)

7. Geisbert, T.W. et al. PLoS Med. 2, e183 (2005).

8. Jahrling, P.B. et al. J. Infect. Dis. 141, 580-589 (1980).

9. Jahrling, P.B., Peters, C.J. \& Stephen, E.L. J. Infect. Dis. 149, 420-427 (1984).

10. Jahrling, P.B. \& Peters, C.J. Infect. Immun. 44, 528-533 (1984).

11. McCormick, J.B. et al. N. Engl. J. Med. 314, 20-26 (1986).

12. Leifer, E., Gocke, D.J. \& Bourne, H. Am. J. Trop. Med. Hyg. 19, 677-679 (1970).

13. Emond, R.T., Bannister, B., Lloyd, G., Southee, T.J. \& Bowen, E.T. Br. Med. J. 285, 1001-1002 (1982).

14. Frame, J.D., Verbrugge, G.P., Gill, R.G. \& Pinneo, L. Trans. R. Soc. Trop. Med Hyg. 78, 319-324 (1984).

15. Cao, W. et al. Science 282, 2079-2081 (1998).

16. Robinson, J.E. et al. Nat. Commun. 7, 11544 (2016).

17. Cross, R.W. et al. Antiviral Res. 133, 218-222 (2016)

18. Thi, E.P. et al. Nature 521, 362-365 (2015).

19. Thi, E.P. et al. Sci. Transl. Med. 6, 250 ra116 (2014). 


\section{ONLINE METHODS}

Study design. 48 healthy adult cynomolgus macaques (Macaca fascicularis) of Chinese origin (3-9 kg) were used to conduct seven separate studies. An initial study employed four untreated control animals to assess the virulence of the seed stock, with 11 controls used in total (Supplementary Table 1, C-1-4). Six successive studies were then performed (including 3-8 treated animals and 1 or 2 controls per study) where the experimental conditions varied (Supplementary Tables 2 and $\mathbf{3}$ ). Group sizes were chosen assuming a one-tailed alpha of 0.05 , with sample size of at least three per group, which provided $>80 \%$ power to detect a difference in the proportion of surviving animals between the treatment group ( $100 \%$ survival rate) and the control group ( $0 \%$ survival rate), using a Fisher's exact test. Animals were randomized, using Microsoft Excel, into treatment or control groups. A number of parameters were monitored during the course of the study, including survival, clinical observations, hematology, serum biochemistry, viremia by RT-qPCR and plaque assay, and tissue pathology. No samples were excluded from analysis in these studies. The overall objective of the study as a whole was to assess protection from LF, with all other measurements considered to be secondary objectives. Study endpoints were predetermined to occur 28-31 d after virus challenge. Data are presented as the means calculated from replicate biological samples, not replicate assays, and error bars represent the s.d. across replicates. This study was not blinded.

Animal challenge. All procedures with live LASV were performed under Biosafety Level 4 maximum containment conditions at the Galveston National Laboratory, Galveston, TX. All animals were inoculated intramuscularly (i.m.) with a target dose of 3,500 PFU of LASV (Josiah strain: Genbank HQ688672 or HQ688674) (actual dose of LASV was 2,250(1), 3,250(2), 3,000(3), 3,625(4), 2,500(5), 4,000(6), and 4,500(7) PFU, from studies 1-7 as indicated, respectively). In the first study, four untreated control animals were used to demonstrate the virulence of the challenge stock. Studies 2-7 were postexposure-treatment studies in which anti-LASV-GP huMAbs were administered to macaques by bolus i.v. infusion. Studies $2-5$ were used to identify the most promising antibodies for preclinical development. In the second study, huMAb 19.7E $(15 \mathrm{mg} / \mathrm{kg})$ was administered to four macaques shortly after LASV challenge (day 0 ) and again on day 5 (Fig. 1a), and one control animal received no treatment. In the third study employing nine macaques, huMAb 37.2D $(15 \mathrm{mg} / \mathrm{kg}$ ) was administered to four macaques shortly after LASV challenge (day 0 ), and again on days 4 and 8 (Fig. 1a), and an equal mixture of huMAb 19.7E and $37.2 \mathrm{D}$ (total dose of $15 \mathrm{mg} / \mathrm{kg}$ ) was administered shortly after LASV challenge (day 0 ) and again on days 4 and 8 (Fig. 1b), whereas one control animal received no treatment. In the fourth study involving ten macaques, huMAb $37.2 \mathrm{D}(6 \mathrm{mg} / \mathrm{kg})$ was administered to two macaques shortly after LASV challenge (day 0) and again on days 4 and 8 (Fig. 1a); huMAb 12.1F $(15 \mathrm{mg} / \mathrm{kg})$ was administered to three animals shortly after LASV challenge (day 0$)$ and again on days 4 and 8 (Fig. 1a); huMAb $37.7 \mathrm{H}(15 \mathrm{mg} / \mathrm{kg})$ was administered to three animals shortly after LASV challenge (day 0 ) and again on days 4 and 8 (Fig. 1a); and two control animals received no treatment. In a fifth study involving nine animals, huMAb $8.9 \mathrm{~F}(15 \mathrm{mg} / \mathrm{kg})$ was administered to four animals shortly after LASV challenge (day 0 ) and again on days 4 and 8 (Fig. 1a); an equal mixture of huMAbs $8.9 \mathrm{~F}, 12.1 \mathrm{~F}$, and $37.2 \mathrm{D}(15 \mathrm{mg} / \mathrm{kg}$ per huMAb) was administered on days 3, 6, and 9 after LASV challenge to four animals (Fig. 1b); and one control animal received no treatment. In a sixth study of five animals, the equal mixture of huMAbs $8.9 \mathrm{~F}, 12.1 \mathrm{~F}$, and $37.2 \mathrm{D}$ ( $15 \mathrm{mg} / \mathrm{kg}$ per huMAb) was administered to four LASV-infected macaques on days 6, 9, and 12 after virus challenge (Fig. 1b); and one control animal received no treatment. In a final study of six animals, the equal mixture of huMAbs $8.9 \mathrm{~F}, 12.1 \mathrm{~F}$, and $37.2 \mathrm{D}(15 \mathrm{mg} / \mathrm{kg}$ per huMAb) was administered to five LASV-infected macaques on days 8, 11, and 14 after virus challenge (Fig. 1b); and one control animal received no treatment. $100 \mu \mathrm{g}$ aliquots of the monoclonal antibodies used in this study are available to verified researchers after execution of a material-transfer agreement with Tulane University and Zalgen Labs. All 48 animals were given physical exams, and blood was collected at the time of challenge and on days $3,5,7$, and 10 (study 1); days $0,3,5,7,10,15$, 22 , and 28 (study 2 ); $0,4,8,11,15,22$, and 30 (study 3 and 4 ); $0,4,8,11,15$, 21 , and 28 or 31 (study 5 ); and $0,6,9,12,15,21$, and 28 (study 6 ); $0,8,11,14$, 21 , and 30 (study 7). In addition, all animals were monitored daily and scored for disease progression using an internal arenavirus scoring protocol approved by the UTMB Institutional Animal Care and Use Committee (IACUC). All IACUC-approved studies performed at UTMB were in compliance with the Animal Welfare Act, PHS Policy and other local and Federal statutes and regulations relating to experiments involving animals. UTMB facilities used in this work are accredited by the Association for Assessment and Accreditation of Laboratory Animal Care International and adhere to principles specified in the eighth edition of the Guide for the Care and Use of Laboratory Animals, National Research Council. The scoring changes measured from baseline included posture and activity level, attitude and behavior, food and water intake, weight, respiration, and disease manifestations, such as visible rash, hemorrhage, ecchymosis, or flushed skin. A score of $\geq 9$ indicated that an animal met the criteria for euthanasia.

Detection of virus load. RNA was isolated from whole blood using the viral RNA mini-kit (Qiagen) using $100 \mu \mathrm{l}$ of blood into $600 \mu \mathrm{l}$ of viral lysis buffer AVL. For tissues (liver, spleen, and brain), approximately $100 \mathrm{mg}$ of each sample was stored in $1 \mathrm{ml}$ of RNAlater (Qiagen) for a minimum of $24 \mathrm{~h}$ to stabilize RNA, and approximately $100 \mathrm{mg}$ was stored for virus isolation. For tissues stored in RNAlater, RNAlater was completely removed and tissues were homogenized in $600 \mu \mathrm{l}$ RLT buffer in a 2-ml cryovial using Qiagen tissue lyser and stainless-steel beads. Primers/probe targeting the NP gene (Genbank HQ688672) of LASV were used for real-time quantitative PCR (RT-qPCR), with the probe used here being 6-carboxyfluorescein (6FAM)- $5^{\prime}$ CCC TCA CTG TGC ACT AAT GGA CTG C3'-6 carboxytetramethylrhodamine (TAMRA) (Life Technologies). LASV RNA was detected using the CFX96 detection system (BioRad Laboratories) in one-step probe RT-qPCR kits (Qiagen) with the following cycle conditions: $50^{\circ} \mathrm{C}$ for $10 \mathrm{~min}, 95^{\circ} \mathrm{C}$ for 10 $\mathrm{s}$, and $40 \mathrm{cycles}$ of $95^{\circ} \mathrm{C}$ for $10 \mathrm{~s}$ and $59^{\circ} \mathrm{C}$ for $30 \mathrm{~s}$. Threshold cycle (CT) values representing LASV S genomes were analyzed with CFX Manager Software, and data are shown as genome equivalents (GEq) (Fig. 2a,c,e). To create the GEq standard, RNA from LASV stocks was extracted, and the number of LASV $S$ genomes was calculated using Avogadro's number, the molecular weight of the LASV genome, and the percentage of the $S$ genome segment to total viral RNA genomes present in the standard sample. Limit of detection was $1 \times 10^{4} \mathrm{GEq} / \mathrm{ml}$.

Virus titration was performed by plaque assay with Vero 76 cells from all serum samples and $10 \%$ weight/volume tissue homogenates (from tissue frozen at $-80^{\circ} \mathrm{C}$ ), as previously described ${ }^{8}$. Briefly, increasing ten-fold dilutions of the samples were adsorbed to Vero 76 monolayers in duplicate wells (200 $\mu \mathrm{l})$; the limit of detection from serum was $15 \mathrm{PFU} / \mathrm{ml}$, and the limit of detection from $10 \%$ tissue homogenates was $50 \mathrm{PFU} / \mathrm{g}$. All cells were verified as mycoplasma free using eMyco-plus mycoplasma detection kit from Boca Scientific.

huMAb-neutralization analysis. Briefly, Vero 76 cells were seeded into six-well plates to generate a confluent monolayer on the day of inoculation. Neutralization assays were performed with $100 \mu \mathrm{g}$ or $50 \mu \mathrm{g}$ of the huMAbs 8.9F, $12.1 \mathrm{~F}, 37.2 \mathrm{D}$, or equal parts of all three in a cocktail prepared in DMEM with $10 \%$ guinea-pig complement and incubated with $\sim 100$ PFU of LASV or LASV $8.9 \mathrm{~F}(\mathrm{e})$ in a total volume of $200 \mu \mathrm{l}$ at $37^{\circ} \mathrm{C}$ for $60 \mathrm{~min}$; controls with no $\mathrm{mAb}$ were included. Medium was removed from cells, the huMAbs-virus mixture was added to duplicate wells, and plates were incubated for $60 \mathrm{~min}$ at $37^{\circ} \mathrm{C}$. The mixture was removed from the cells and $2 \mathrm{ml}$ of $0.9 \%$ agarose in EMEM with 5\% FBS were overlaid onto each of the wells. Cells were observed 2-4 d postinoculation, and plaques were visualized and counted using neutral red stain.

Binding of 8.9F, 12.1F, and 37.2D to LASV-GP-Y150D expressing cell lines. The specific binding of each huMAb component was assayed on cell lines engineered to express LASV GPC transiently. The complete open reading frame (ORF) of the LASV glycoprotein was cloned in the pcDNA3.1 + intA vector, verified by Sanger DNA sequencing, and transfected in HEK-293T/17 cells at $\sim 70 \%$ confluency in poly-D-lysine-coated six-well polystyrene cell-culture plates. Transfection was performed with polyethylenimine (PEI)-based reagent. $24 \mathrm{~h}$ post-transfection, cells were washed once with $1 \times \mathrm{PBS}, \mathrm{pH} 7.4$ and incubated with fresh $1 \times$ PBS, pH 7.4, until cells detached. Cells were gently lifted with a cell-culture scrapper and gently resuspended by pipetting to generate 
a dispersed cell suspension. Cells were pelleted by low-speed centrifugation (200g) and resuspended in FACS buffer (1× PBS, pH 7.4/2\% fetal bovine serum (FBS) $/ 0.05 \%$ sodium azide). LASV-GPC-expressing or mock-transfected cell lines were incubated for 20 min on ice in FACS buffer containing each LASV huMAb or an isotype control antibody. Following washing steps, the cells were incubated with a goat anti-human $\operatorname{IgG}(\mathrm{H}+\mathrm{L})$-AlexaFluor-488-labeled secondary antibody diluted in FACS buffer, on ice, for $20 \mathrm{~min}$. Cells were then washed and resuspended in FACS buffer containing $1 \mathrm{mg} / \mathrm{ml}$ propidium iodide, a viability dye that permits the discrimination of live and dead cells. Cells were analyzed by FACS using an Accuri C6 Flow Cytometer.

Deep-sequence analysis of RNA genome of LASV 8.9F-MAb escape mutant. To analyze the potential for a virus escape mutant, virus was isolated $(1 \times$ passage on Vero E6) from the 21-d postchallenge sample isolated from animal STx-17. Briefly, viral RNA was isolated from a Trizol LS (Invitrogen)/sample mixture using a Direct-zol RNA mini-prep (Zymo Research), per the manufacturer's instructions. Approximately $150 \mathrm{ng}$ of purified RNA was used to make cDNA using the Ovation RNA-seq 2.0 kit (NuGen), and this, in turn, was used for the preparation of the double-stranded DNA library, using Encore Ion Torrent library prep kit. Sequencing was performed by the UTMB Molecular Core on the Ion Torrent using 318-v2 deep-sequencing chips. Sequence analysis was performed using DNA Star Seqman NGen software (DNA Star) on the basis of unpaired analysis of 125-bp overlaps.

Detection of antibodies against LASV proteins. Enzyme-linked immunosorbent assays (ELISA) using recombinant LASV proteins (NP; Genbank HQ688672), which were optimized for the detection of virus-specific IgM and IgG levels in serum, were coated in strip-well plates, blocked, dried, and packaged with desiccating packs (Zalgen Labs; 10004 ReLASVTM Pan-Lassa IgG/IgM ELISA Test Kit) ${ }^{20}$. For analysis, primate sera were diluted 1:100 in a proprietary-sample dilution buffer and incubated in wells for $30 \mathrm{~min}$ at room temperature, washed, and then incubated with a 1:2,500 dilution of HRPlabeled anti-monkey IgG (Fitzgerald Industries International, 43R-IG020HRP) for an additional $30 \mathrm{~min}$. After washing, detection was performed with tetramethylbenzidine substrate for $10 \mathrm{~min}$ at room temperature, stopped with sulfuric acid, and then read at an absorbance of $450 \mathrm{~nm}$ using a Molecular Devices ELISA plate reader. Reciprocal titers were calculated using mean +3 s.d. cutoffs established with similarly diluted baseline serum controls.

Hematology and serum biochemistry. Total white blood cell counts, white blood cell differentials, red blood cell counts, platelet counts, hematocrit values, total hemoglobin concentrations, mean cell volumes, mean corpuscular volumes, and mean corpuscular hemoglobin concentrations were analyzed from blood collected in tubes containing EDTA using a laser-based hematologic analyzer (Beckman Coulter). Serum samples were tested for concentrations of albumin, amylase, alanine aminotransferase (ALT), aspartate aminotransferase (AST), alkaline phosphatase (ALP), gamma-glutamyltransferase (GGT), glucose, calcium, uric acid, total protein, blood urea nitrogen (BUN), creatinine (CRE), and C-reactive protein (CRP) by using a Piccolo point-of-care analyzer and Biochemistry Panel Plus analyzer discs (Abaxis).

Histopathology and immunohistochemistry. Necropsy was performed on all subjects. Tissue samples of all major organs were collected for histopathologic and immunohistochemical examination, immersion-fixed in 10\% neutral-buffered formalin, and processed for histopathology, as previously described. For immunohistochemistry, specific anti-LASV immunoreactivity was detected using either an anti-LASV GP or an anti-LASV Z protein rabbit primary antibody (Integrated BioTherapeutics) at a 1:1,000 dilution for the GP antibody and a 1:500 dilution for the anti- $Z$ antibody. In brief, tissue sections were processed for immunohistochemistry using the Dako Autostainer (Dako). Secondary antibody used was biotinylated goat anti-rabbit IgG (Vector Laboratories) at 1:200 followed by Dako LSAB2 streptavidin-HRP (Dako). Slides were developed with Dako DAB chromagen (Dako) and counterstained with hematoxylin. Nonimmune rabbit IgG was used as a negative control.

Data availability. All data are available from the corresponding authors upon reasonable request. Further methods information can be found in the Life Sciences Reporting Summary.

20. Grove, J.N. et al. Virol. J. 8, 314-314 (2011). 


\section{nature research}

Thomas W. Geisbert

Corresponding author(s): Robert F. Garry

Initial submission $\bigotimes$ Revised version

Final submission

\section{Life Sciences Reporting Summary}

Nature Research wishes to improve the reproducibility of the work that we publish. This form is intended for publication with all accepted life science papers and provides structure for consistency and transparency in reporting. Every life science submission will use this form; some list items might not apply to an individual manuscript, but all fields must be completed for clarity.

For further information on the points included in this form, see Reporting Life Sciences Research. For further information on Nature Research policies, including our data availability policy, see Authors \& Referees and the Editorial Policy Checklist.

\section{- Experimental design}

\section{Sample size}

Describe how sample size was determined.

\section{Data exclusions}

Describe any data exclusions.

\section{Replication}

Describe whether the experimental findings were reliably reproduced.

\section{Randomization}

Describe how samples/organisms/participants were allocated into experimental groups.

\section{Blinding}

Describe whether the investigators were blinded to group allocation during data collection and/or analysis.
Group sizes were chosen assuming a one-tailed alpha of 0.05 , with sample size of at least 3 per group providing $>80 \%$ power to detect a difference in proportion of surviving animals between the treatment group (100\% survival rate) and the control group ( $0 \%$ survival rate), using a Fisher's exact test.

No data were excluded from the analyses.

Seven separate nonhuman primate studies were performed. No study was an exact repeat of a prior study. However, the separate challenge studies were performed in an iterative manner with a similar experimental design where the time of initiation of monoclonal antibody treatment was increasingly delayed to determine how late in the disease course the antibody cocktail could be delivered and still be protective.

Animals were randomized with Microsoft Excel into treatment or control groups.

The studies were not blinded as animals were treated with monoclonal antibodies intravenously in a Biosafety Level (BSL)-4 laboratory. As these studies were performed as proof of concept studies (rather than pivotal studies for licensure under the US FDA Animal Rule) in a high containment BSL-4 environment the control animals were untreated to reduce risk to the work force to the maximum extent possible.

Note: all studies involving animals and/or human research participants must disclose whether blinding and randomization were used. 


\section{Statistical parameters}

For all figures and tables that use statistical methods, confirm that the following items are present in relevant figure legends (or in the Methods section if additional space is needed).

$\mathrm{n} / \mathrm{a} \mid$ Confirmed

The exact sample size ( $n$ ) for each experimental group/condition, given as a discrete number and unit of measurement (animals, litters, cultures, etc.)

$\triangle$ A description of how samples were collected, noting whether measurements were taken from distinct samples or whether the same sample was measured repeatedly

$\bigotimes$ A statement indicating how many times each experiment was replicated

The statistical test(s) used and whether they are one- or two-sided (note: only common tests should be described solely by name; more complex techniques should be described in the Methods section)

A description of any assumptions or corrections, such as an adjustment for multiple comparisons

$\bigotimes$ The test results (e.g. $P$ values) given as exact values whenever possible and with confidence intervals noted

A clear description of statistics including central tendency (e.g. median, mean) and variation (e.g. standard deviation, interquartile range)

Clearly defined error bars

See the web collection on statistics for biologists for further resources and guidance.

\section{- Software}

Policy information about availability of computer code

\section{Software}

Describe the software used to analyze the data in this study.

GraphPad Prism 7 was used to analyze the Kaplan-Meier curves by Mantel-Cox test.

For manuscripts utilizing custom algorithms or software that are central to the paper but not yet described in the published literature, software must be made available to editors and reviewers upon request. We strongly encourage code deposition in a community repository (e.g. GitHub). Nature Methods guidance for providing algorithms and software for publication provides further information on this topic.

\section{- Materials and reagents}

Policy information about availability of materials

\section{Materials availability}

Indicate whether there are restrictions on availability of unique materials or if these materials are only available for distribution by a for-profit company.

\section{Antibodies}

Describe the antibodies used and how they were validated for use in the system under study (i.e. assay and species).

\section{Eukaryotic cell lines}

a. State the source of each eukaryotic cell line used.

b. Describe the method of cell line authentication used.

c. Report whether the cell lines were tested for mycoplasma contamination.

d. If any of the cell lines used are listed in the database of commonly misidentified cell lines maintained by ICLAC, provide a scientific rationale for their use.
$100 \mu \mathrm{g}$ aliquots of the monoclonal antibodies used in this study are available to verified researchers after execution of a material transfer agreement with Tulane University and Zalgen Labs

Validation of each antibody was performed by sequence analysis of antibody heavy and light-chain constructs. Sequence accuracy was verified using multiple independent clones for each unique heavy and light chain. Specificity of each antibody was tested by flow cytometry on cell surface-expressed arenaviral glycoproteins and on control filoviral glycoproteins. The antibodies were previously described and used in the studies outlined in Cross et al. Antiviral Res 133, 218-222, doi:10.1016/j.antiviral.2016.08.012(2016).

VERO C1008 [Vero 76, clone E6, Vero E6] (ATCC ${ }^{\circledR}$ CRL-1586 ${ }^{\mathrm{TM}}$ ) were purchased from ATCC (Manassas, VA, USA).

A Certificate of Analysis was provided by ATCC.

The cell lines were negative for mycoplasma.

No commonly misidentified cells lines were used. 
Policy information about studies involving animals; when reporting animal research, follow the ARRIVE guidelines

11. Description of research animals

Provide details on animals and/or animal-derived materials used in the study.

Forty-eight healthy adult cynomolgus macaques (Macaca fascicularis) of Chinese origin (3-9 kg) were used to conduct seven separate studies. Both males and females were employed as shown in Supplementary Tables 1 and 2.

Policy information about studies involving human research participants

\section{Description of human research participants}

Describe the covariate-relevant population characteristics of the human research participants.

The study did not involve human research participants. 